\title{
Collaborate to Innovate: Utilizing Design Patterns Cards for Accelerating the Digital Transformation of Small and Medium-sized Enterprises
}

\author{
Christoph Gerling \\ Humboldt Institute f. Internet \\ and Society, Germany \\ christoph.gerling@hiig.de bickel.fabrice@hotmail.com
}

\author{
Thomas Haskamp \\ Hasso Plattner Institute, \\ Germany \\ thomas.haskamp@hpi.de
}

\author{
Falk Uebernickel \\ Hasso Plattner Institute, \\ Germany \\ falk.uebernickel@hpi.de
}

\begin{abstract}
As more and more organizations reach the limits of their internal capabilities to deal with the challenges induced by digital transformation, they are increasingly forced to seek external digitalization opportunities. In particular, small and medium enterprises are affected by this due to their limited human and financial resources. Currently, there is a lack of overview of options considering limited internal digital capabilities and resources. Thus, we choose an action design research approach to develop an external digitalization activity navigator. As a result, we derive five design principles for successful navigation and 30 activities, which are presented as design pattern cards. Our work can help practitioners and scholars alike to structure external digitalization activities.
\end{abstract}

\section{Introduction}

In recent years, digital transformation (DT) has disrupted whole industries such as automotive [1], telecommunication [2], or media industry [3]. The transformation is a major organizational change induced by digital technologies [4]. As part of the transformation, organizations must rethink, adjust, and innovate their processes, products, services, and business models [5]. In doing so, they must embrace new digital technologies and adapt their strategy, organizational structure, and culture [6]. They are not only forced to deal with new technologies but also with new competitors from other industries, with different business models [7]. This disruption is reflected as both an existential risk and a rewarding opportunity. Mastering DT is a key factor in surviving or even profiting from digital disruption [8].

While coping with the challenges of DT, large enterprises can develop and deploy their digital technologies and ecosystems [9]. Equipped with numerous resources and capabilities, they can orchestrate internal resources to build critical capabilities that enable them to overcome organizational inertia and resistance to change [10]. In contrast, most small and medium enterprises (SMEs) lack sufficient digital capabilities and resources [11]. DT imposes daunting challenges on SMEs [12], ranging from assessing digital technology needs and contingencies [13] over missing capabilities required to manage new digital technologies and business models [14] to the consideration of growing complexity in the ecosystem [15]. Therefore, most SMEs rely on digital capabilities from external partners [16].

Although there is a sense of urgency to respond to DT, many SMEs do not know how to adapt and cope with disruption [17]. In particular, there is a lack of overview of how external capabilities, skills, and knowledge can be integrated to get started [18]-[20]. While extant research already covers many single activities such as creative pilot projects [21], hackathons [22], or co-creation activities [23], there is a lack of navigational assistance. To address this shortcoming, our goal is to develop and collect practice-oriented design knowledge from a navigational and action-taking perspective. We, therefore, develop a holistic and concise navigator for external digitalization activities (EDAs) in practice. The intersection between the need to provide an overview of EDAs and the gap in the scientific knowledge base defines the problem space of interest for this study [24], leading us to formulate the following research question: What are the design principles for a navigation assistant that helps SMEs maneuver successfully through existing external digitization activities?

To answer the research question, we follow an action design research (ADR) approach [25] to develop an artifact in form of an EDA navigator. Thereby, we consider the bodies of digital transformation and open innovation literature and theory of design patterns and principles. Following van Aken [26], applying the concept of design pattern cards, we identify (1) a set of relevant design principles for the actual card layout (object design) and (2) a set of design principles for the utilization of the cards (realization design). Further, we demonstrate how design pattern cards can be designed and implemented in a real-world context. Thereby, we draw on 
ten preliminary interviews, ten in-depth interviews with experts and SME representatives, and eight evaluating interviews with SME managers. Hence, we contribute to the domain of DT of SMEs and show how they can generate new knowledge about DT-related open innovation activities. From a managerial perspective, this study can guide practitioners on how to use design patterns to facilitate digitalization in SMEs utilizing EDAs.

\section{Conceptional Background}

\subsection{Digital Transformation by SME}

Digital transformation (DT) is seen as a "process that aims to improve an entity by triggering significant changes to its properties through combinations of information, computing, communication, and connectivity technologies" [6, p. 121]. In contrast to digitization, which focuses on standardizing and automating processes to reduce costs, DT changes the value proposition and structure of the organization. The cardinal changes within the firm's products, services, and business models are also referred to as "digitalization" [8]. For this reason, the terms "digitization" and "digital transformation" are largely used interchangeably in this paper.

In contrast to the digitalization success stories (e.g., WeChat, Airbnb, or Netflix), small and medium-sized enterprises (SMEs) face different framing conditions on their way to DT. While larger firms are beginning to understand the opportunities and challenges of DT, SMEs still struggle and encounter challenges when trying to innovate [11]. Their level of digitalization is still below the industry average. According to Pelletier and Cloutier [12], hurdles to successful DT in SMEs are threefold. First, digitization efforts are often hindered because SME entrepreneurs and managers lack an overarching strategy and cannot prioritize their key digitalization challenges [27]. In particular, there is a shortage of appropriate support in that area. Second, SMEs frequently lack necessary technological capabilities such as technological knowledge, skills of employees, and advanced technologies [28]. Third, SMEs occasionally lack expertise when operating in digital ecosystems [29]. This involves building and managing relationships with digital technology specialists and service providers, as well as a missing collaborative, sharing culture [15], [27].

There are numerous contributions to activities that help SMEs overcome these hurdles and successfully manage DT. E.g., changing the organizational structure by introducing a chief digital officer is reflected as helpful [30]. In general, digitalization activities emerge in various forms. In line with Barthel et al. [31], we define digitalization activities as a measure to achieve a valueadding solution enabled by digital technology, marked by (perceived) novelty in its technical or organizational component, and contributes to a firm's DT efforts. Digitalization activities are characterized by "relatively low controllability and transparency, have heterogeneous stakeholders that cooperate in novel combinations, and can create different value contributions in a variety of manners that are often difficult to predict" [31, p. 4]. As a result, digitalization is not a well-bounded phenomenon focused on fixed products, services, or organizations. There is a shift toward less predefined and more distributed business activity. Hereafter, we discuss how opening up SMEs can help them embrace DT to access resources and skills they do not possess internally.

\subsection{Open Innovation by SME}

Current research indicates that innovation can take place in two ways, within or beyond the boundary of a firm [32]. First, firms can take an internal approach to innovation, where they innovate by acquiring, processing, integrating, and leveraging internal knowledge and resources. Second, learning from partners or business relationships and tapping knowledge from the external environment can be a source of innovation [32]. Firms can thus adopt an external approach to innovation by acquiring and processing knowledge and resources from external partners and integrating them with their knowledge to strive for innovation. In line with Chesbrough et al., we define open innovation as "the use of purposive inflows and outflows of knowledge to accelerate internal innovation and expand the markets for external use of innovation, respectively" [33, p. 2].

In this study, we focus on firms' open search behavior for innovation outcomes to drive digitization, i.e., seeking external actors and sources to help them achieve and sustain DT. Rather than creating new businesses to advance their digital technologies, capabilities, and innovation-enabling culture, SMEs can significantly reduce costs by collaborating with companies that already possess these assets. In particular, collaborating with young technology ventures seems promising, as they generally take an open innovation approach and are normally composed of younger employees with up-to-date skills and technical knowledge [34]. Such collaborations are a potential approach to solve the challenges of SMEs' DT. Therefore, this study focuses on activities from the SME of external search within the inbound open innovation approach, not excluding coupled processes. Thus, we define external digitalization activities (EDAs) as the extent of measures to which a firm opens up to collaborate to innovate with its external constituents such as market leaders, suppliers, competitors, and clients regarding the exploitation of digital technology. The activities considered here that comply with this definition are shown in Figure 1 based on Wrobel et al. [35] and clustered according to their collaboration intensity 
and time horizon. Further activities were added as part of the ADR approach based on expert knowledge.

In summary, EDAs are "iterative, fractal, and chaotic" [36, p. 56]. They typically involve short learning cycles, the outcomes of the activities do not always have predictable market potential, and the exact use is not necessarily predetermined. Even in more advanced digitization activities, the generativity of digital innovation leads to openness [37]. EDAs show a strong focus on experimentation, value creation, and partner integration [38]. Further, expectations for outcomes often vary and sometimes include unclear ideas about how to promote long-term organizational change. Overall, organizations may implement EDAs in pursuit of a wide variety of goals, as digital innovations enable very different value propositions. SMEs can learn new ways to reconfigure existing knowledge allocation and use it for innovation [33]. In the following, the EDAs are considered as design patterns and depicted on cards. The corresponding design principles need to be explored.

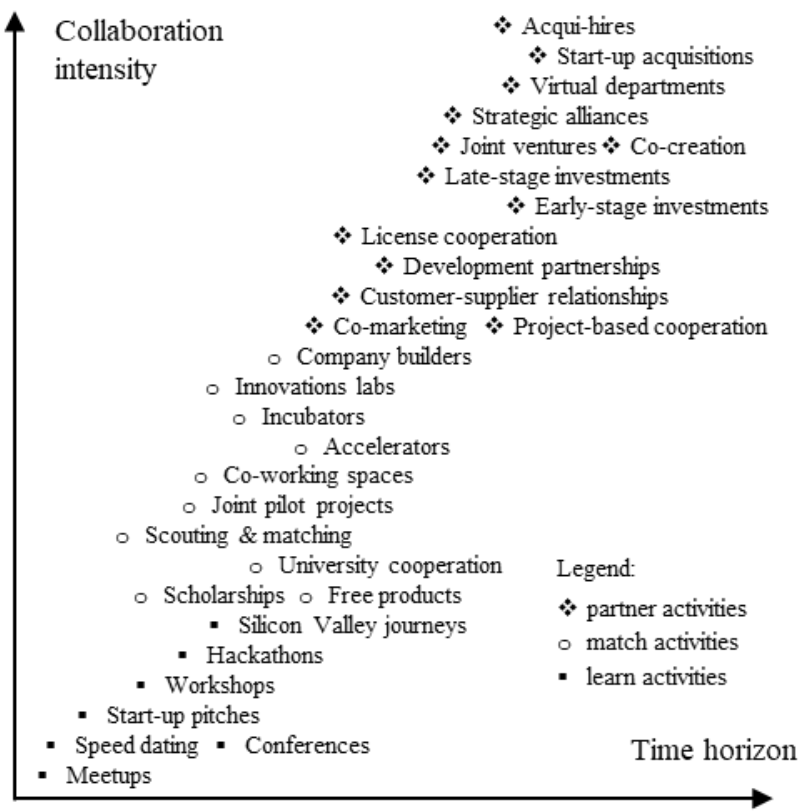

Figure 1: External digitalization activities [35]

\section{Research Methodology}

Our project started in January 2020 and ended in February 2021. The goal was to develop design pattern cards and principles for initiating external digitization activities. A pattern generally describes a recurring problem and the core of the solution to that problem, so that the solution can be used millions of times without having to solve the problem twice [39]. Because design patterns are reusable, they increase the efficiency of a design process and the effectiveness of the solution, since they have already proven useful in a given context. Further, design patterns help create a common language and allow users to think about their use in different contexts [40]. Patterns are developed based on experience and observations providing a tool to transform tacit knowledge into explicit one. For simplicity and clarity, design patterns must follow the same format [41].

In addition to their application in architecture [41] and software engineering [42], design patterns are also used in management [43]. In business modeling, design patterns support the analysis of the logic of business model designs and the communication of archetypes [44]. Since previous research in business model innovation has shown that artifacts such as design pattern cards can significantly help managers in their problem analysis, idea generation, and result evaluation [45], this study intends to take advantage of such design patterns by applying them to the field of EDAs. In this respect, the contribution of this research can be seen as an adaptation of known solutions to new problems [46]. Thereby, we incorporate von Aken's distinction between the two types of designs: "an object-design, the design of the intervention or the artifact [and] a realization-design, i.e., the plan for the implementation of the intervention" [26, p. 226]. Thus, in terms of our research, the object design refers to the EDA design pattern represented as easy to handle card and the realization design is the organizational context, in which the pattern cards are used, in our case, a workshop format accessible to SMEs. Thereby, design principles help synthesize and formalize design knowledge. Design principles are "prescriptive statements that show how to do something to achieve a goal" [47, p. 1623]. The object design falls into the category of design principles "about an artifact, which focuses on the features that should be built into an artifact, including shape/architecture and function" [47, p. 1634]. The realization design belongs to the category of design principles that "state what (human) users should be able to do with an artifact" [47, p. 1634].

To derive our design principles, we relied on the action design research (ADR) method [26]. This decision was based on the fact that ADR combines the strengths of design research, developing in essence innovative and useful solutions for classes of problems that are relevant to practice [25]. The ADR approach recognizes the importance of collaborating directly and closely with experts and practitioners to ensure the possibility to learn from the intervention in an organizational context to iteratively improve the design artifacts. As a result, we follow Sein et al.'s [26] conceptualization of the artifact as an ensemble artifact, meaning that the artifact includes dimensions beyond the technical aspect. Further, we chose to use circular design to iteratively evaluate and improve the design principles. Consequently, ADR is the most suitable method to achieve our goal, 
since we aim to develop design principles that support SMEs in initializing EDAs. The ADR method consists of four stages which are presented hereafter.

Stage 1: Problem Formulation. We formed an ADR team consisting of two researchers and four experts from training providers who had worked with the researchers in previous projects (experience range from 3 to 12 years). In the first step, we interviewed three SME managers (accounting, personnel services, and manufacturing) about their challenges in digitalization and had them describe their work reality focusing on collaborations to capture a sense of the field conditions. Then, we conducted seven individual interviews with experts in the field of corporate incubation and collaboration (ranging from university to in-house incubators) and validated the findings together with all interviewees in a shared workshop. Based on the preliminary study, we identify three problems/hurdles the SMEs were facing in the context of DT (see 4.1 ). Table 1 provides an overview of the methods used and the output generated in the problem formulation stage (and following stages).

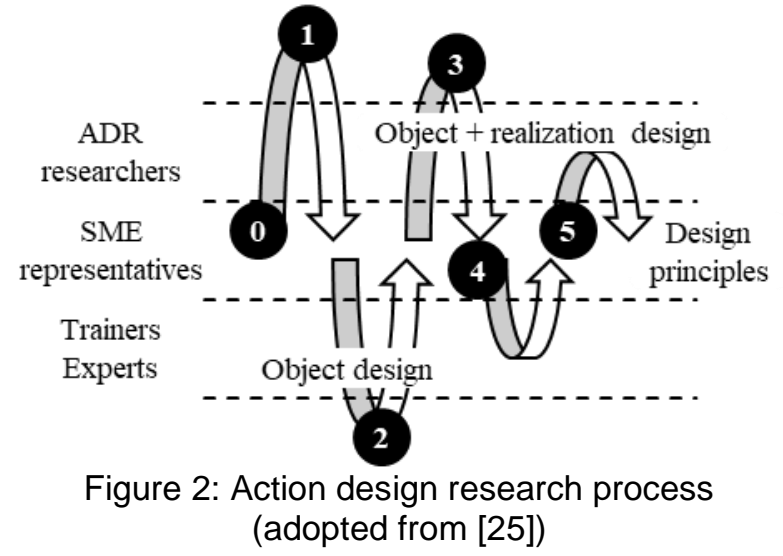

Stage 2: Building, Intervention, and Evaluation (BIE). As the problems were related to organizational structures and processes as a whole as well as the design artifact, we followed an organizational dominant BIE [25]. Figure 2 shows a graphical illustration of the BIE in the design journey. During our design journey, we applied different research methods in the BIE stage. We initially examined the existing literature on the three identified problems/hurdles, using search strings such as "SME digitalization success", "SME open innovation", or "SME collaboration", querying the databases "Science Direct", "AIS eLibrary", and "IEEE" to identify the initial design principles. More specifically, we collected design recommendations that were categorized by the ADR team and thereby identified seven literature issues. On this basis, we have created the first version of pattern cards. Thereafter, we relied on semistructured interviews to evaluate and refine the different versions of our design pattern cards and principles, as we wanted to generate rich insights into the strengths and weaknesses from the experts' perspective. For the comprehensibility of our research process, Table 1 provides details of the methods used, including inputs and outputs. The numbers shown in Figure 2 refer to the numbers in the first column in Table 2. More details about each stage are presented in Section 4.

Table 1: Problem formulation and BIE process including inputs, methods used, and outputs

\begin{tabular}{|c|c|c|c|}
\hline \multicolumn{2}{|c|}{\begin{tabular}{l|l} 
Part Input(s) \\
\end{tabular}} & Method(s) used & Output(s) \\
\hline \multicolumn{4}{|c|}{ Problem formulation stage } \\
\hline 0 & $\begin{array}{l}\text { Project } \\
\text { initialization }\end{array}$ & $\begin{array}{l}\text { - } 3 \text { open interviews } \\
\text { with SME managers } \\
\text { - } 7 \text { semi-structured } \\
\text { interviews with train- } \\
\text { ing providers }\end{array}$ & $\begin{array}{l}3 \text { problems of SME } \\
\text { facing digitalization }\end{array}$ \\
\hline \multicolumn{4}{|c|}{ BIE process stage } \\
\hline 1 & $\begin{array}{l}3 \text { problems from for- } \\
\text { mulation stage }\end{array}$ & $\begin{array}{l}\text { - Literature review } \\
\text { - } 7 \text { semi-structured } \\
\text { inter-views with } \\
\text { training providers } \\
\text { - Drafting design pat- } \\
\text { tern cards }\end{array}$ & $\begin{array}{l}\text { - Set of literature is- } \\
\text { sues (LIs) } \\
\text { - Initial set of design } \\
\text { pattern cards }\end{array}$ \\
\hline 2 & $\begin{array}{l}\text { Initial set of design } \\
\text { pattern cards }\end{array}$ & $\begin{array}{l}5 \text { semi-structured in- } \\
\text { terviews with experts }\end{array}$ & $\begin{array}{l}\text { - Set of expert re- } \\
\text { quirements (ERs) } \\
\text { - Feedback focusing } \\
\text { on the completeness } \\
\text { and understandability } \\
\text { and the user activities }\end{array}$ \\
\hline 3 & $\begin{array}{l}\text { Feedback focusing on } \\
\text { the completeness and } \\
\text { understandability and } \\
\text { the user activities }\end{array}$ & $\begin{array}{l}\text { - Redesign of design } \\
\text { pattern cards (object } \\
\text { design) } \\
\text { - Drafting of work- } \\
\text { shop format (realiza- } \\
\text { tion design) }\end{array}$ & $\begin{array}{l}\text { - Object design and } \\
\text { meta-requirements } \\
\text { (MRs) } \\
\text { - Realization design } \\
\text { and meta-require- } \\
\text { ments (MRs) }\end{array}$ \\
\hline 4 & $\begin{array}{l}\text { - Object design and } \\
\text { MRs about the arti- } \\
\text { fact } \\
\text { - Realization design } \\
\text { and MRs about the } \\
\text { user activity }\end{array}$ & $\begin{array}{l}\text { - } 5 \text { semi-structured } \\
\text { interviews with SME } \\
\text { representatives in a } \\
\text { focus group setup } \\
\text { - Redesign of work- } \\
\text { shop format (object } \\
\text { design) }\end{array}$ & $\begin{array}{l}\text { - Initial set of design } \\
\text { principles (DPs) } \\
\text { - Feedback focusing } \\
\text { on understandability, } \\
\text { helpfulness, and ac- } \\
\text { tionability }\end{array}$ \\
\hline 5 & $\begin{array}{l}\text { - Refined object de- } \\
\text { sign and DPs about } \\
\text { the artifact } \\
\text { - Refined realization } \\
\text { design and DPs about } \\
\text { the user activity }\end{array}$ & $\begin{array}{l}\text { - } 8 \text { semi-structured } \\
\text { interviews with SME } \\
\text { representatives } \\
\text { - Iterative refinement } \\
\text { of design principles } \\
\text { within the ADR team }\end{array}$ & $\begin{array}{l}\text { Final set of number } \\
\text { design principles }\end{array}$ \\
\hline
\end{tabular}

Stage 3: Reflection and Learning. Throughout the 13-month project, especially after single BIE parts, we reflected on the results generated and incorporated additional insights from practice and literature to ensure that our design pattern cards and principles were similar to a solution not for our participants' problem, but a broader class of problems, in our case, providing a navigator for EDAs. For this purpose, there we conducted three group discussions within the ADR team. We believe our project setting helped achieve this goal as we worked with a variety of SMEs, ranging from manufactures to service providers, rather than just one.

Stage 4: Formalization of Learning. To ensure that our results are ready to use for both practitioners and researchers, we derived a total set of 30 design pattern cards and formulated five related design principles following [47]. Addressing the development phase, we 
created and evaluated two versions of the object and realization design within two BIE cycles (see Figure 2 and Table 1, steps 1 to 5) until coming up with a final version. For this purpose, we derived a set of meta-requirements (MRs) in the BIE phase based on the current issues of the scientific literature (LIs) and the requirements of experts (ERs). The design principles (DPs) we developed addressing the corresponding MRs. The DPs in turn form the foundation of the design features (DFs) of the design pattern cards. Our design procedure is elaborated in detail in the following.

\section{Design and Development}

\subsection{Problem Formulation}

The first stage of the ADR approach [25] involves problem formulation. The problem is described by the three hurdles in the DT of SMEs that we specified in more detail in a preliminary study with three SME managers and four training providers (in total ten ethnographical interviews) based on the hurdle described previously in the conceptional background: (1) SME managers lack an overarching strategy and overview to prioritize needs, (2) SMEs lack adequate technical skills and entrepreneurial spirit, and (3) SMEs lack a collaborative culture to establish and manage relationships with digital technology entrepreneurs and service providers taking into account the increasing complexity of a digital ecosystem. These hurdles constituted the starting point for the development of the design pattern cards and principles within our ADR project.

\subsection{Building, Intervention, Evaluation}

The problem formulation (stage 1) serves as the foundation for deriving the requirements from (a) literature and (b) ten semi-structured interviews with experts and practitioners alike (five interviews each) according to Gläser and Laudel [49]. In the following, we will describe and discuss how we gathered the (meta-)requirements and derived the design principles as well as features, relevant for the development of the design pattern cards. The main insights are illustrated in Figure 3.

Deriving Requirements from Literature. We gathered requirements from literature fundamental to the development of the EDA navigator. We identified three broad areas for deriving requirements: SME digitalization success, SME open innovation, and SME collaboration success. Further, we have summarized similar topics as literature issues (LIs) and formed from clusters from them, meta-requirements (MRs). As a result, we obtained three meta-requirements for the object and two for the realization design.

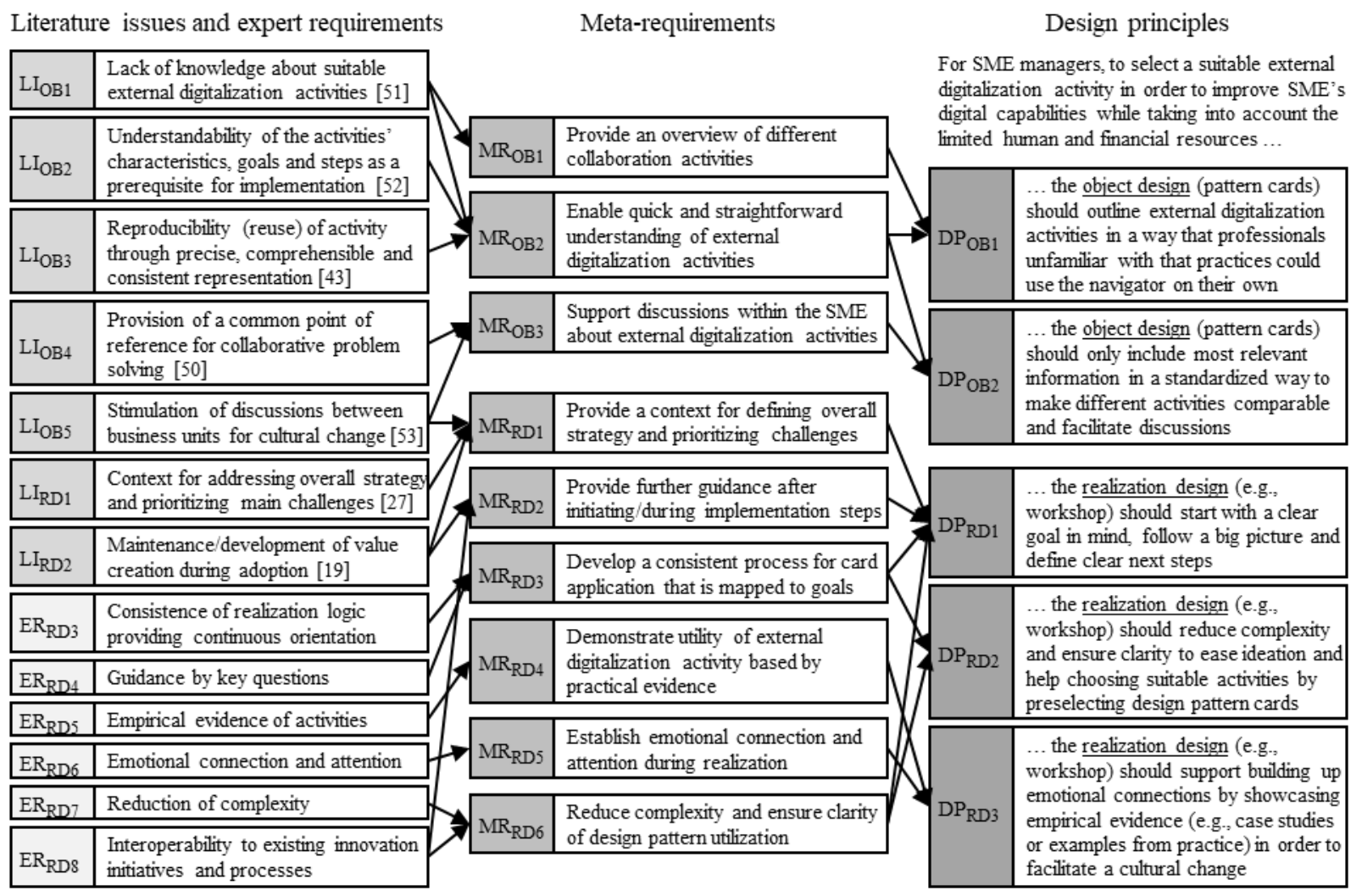

Figure 3: Overview of derived design principles according to Gregor et al. [47] 
The first object meta-requirement $\left(\mathrm{OD}_{\mathrm{MR} 1}\right)$ deals with providing an overview of different collaboration activities. This addresses the problem of lacking knowledge of SME managers about possible activities for unlocking DT unlocking [51], [52]. The second object meta-requirement $\left(\mathrm{OD}_{\mathrm{MR} 2}\right)$ focuses on enabling a quick and straightforward understanding of EDAs. Decision-makers who are inexperienced with collaborations not only need an overview of the activities, but must also be able to understand their characteristics, goals, and steps [51], [52]. Therefore, design patterns must be precise, comprehensible, and consistent [43]. The third object meta-requirement $\left(\mathrm{OD}_{\mathrm{MR} 3}\right)$ states to support discussions in SMEs on the EDAs. To build effective collaborations, SME managers from different business units need to actively discuss the potential benefits and pitfalls of different activities [53]. The design patterns must therefore serve as a stimulus for discussion. We now turn to the realization design. The first realization meta-requirement $\left(\mathrm{RD}_{\mathrm{MR} 1}\right)$ calls for providing a context for defining the overall strategy and prioritizing challenges. Since digitization projects are mostly hindered because managers lack an overall strategy and cannot prioritize their main challenges, a context must be provided that addresses these issues [27]. Further, the second meta-requirement ( $\left.\mathrm{RD}_{\mathrm{MR} 2}\right)$ considers that further guidance is needed after the initiation of the activity / during the implementation steps. The solution should help maintain value creation during adoption processes and be useful after initiation phases [19], [20].

Deriving Requirements from Expert Interviews. After defining a set of literature-based meta-requirements, we identified requirements originating from specific field problems regarding the realization design, i.e., the user activities of the SME decisionmakers to be supported. This expands the first two realization design meta-requirements by another four. Thus, the third realization meta-requirement $\left(\mathrm{OD}_{\mathrm{MR} 4}\right)$ reflects a consistent process for pattern card utilization that is aligned with goals. The realization design (i.e., workshop) must be consistent and map to the predefined goals, even though the process will likely be different for each SME. To achieve this, the process should be guided by structured key questions. The fourth realization meta-requirement $\left(\mathrm{OD}_{\mathrm{MR} 4}\right)$ demands demonstrating the benefits of EDAs based on practical evidence. It is critical to showcase the benefits of the activities based on actual evidence from SMEs that have already initiated such collaborative activities, e.g., through case studies or real-world examples. The fifth realization meta-requirement $\left(\mathrm{OD}_{\mathrm{MR} 5}\right)$ refers to establishing an emotional connection and attention. The implementation design should include tasks that demonstrate how important DT is in the current market reality, as employees who are emotionally affected are more attentive during workshops. Workshop methods commonly used in start-ups can demonstrate the innovative and fast-paced setting of DT. The final realization meta-requirement $\left(\mathrm{OD}_{\mathrm{MR} 6}\right.$ ) calls for keeping complexity low and ensuring clarity in the design pattern utilization. The structure of the implementation design should be supported by known tools and ensure a preselection of pattern cards relevant to the specific challenges of the participants (e.g., through a decision tree). Furthermore, it should be possible to use the cards during a firm's ongoing innovation process.

Deriving Design Principles and Features. Based on our meta-requirements, we established a set of design principles (DPs), as shown in Figure 3. We instantiated the five final DPs through 15 design features (DFs) in, for now, the final version of our EDA navigator, guided by Gregor et al. [47]. We start with the object design-related principles demonstrated by the example of a pattern card in Figure 4.

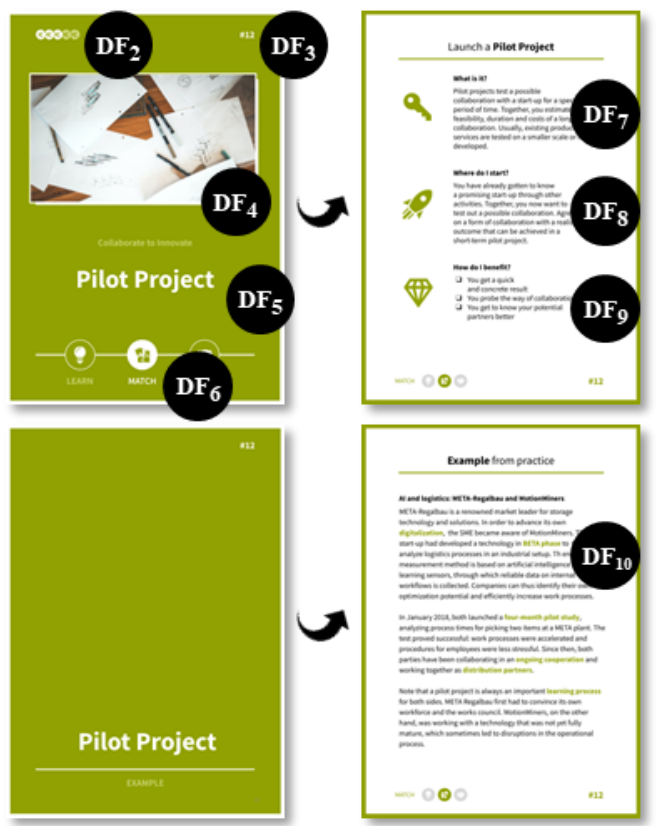

Figure 4: Example of design pattern card

The first object design principle $\left(D P_{O B I}\right)$ specifies that the pattern cards should be presented in a way SME managers unfamiliar with EDAs could use the navigator on their own. Therefore, we added two explanatory cards. One of the cards contains an overview of all activities clustered by the phases learn, match, and partner $\left(\mathrm{DF}_{1 *}\right)$ based on Figure 1 . The asterisk indicates that the features are not explicitly displayed on figures here. Further, we have attempted to keep the descriptions as simple and self-explanatory as possible $\left(\mathrm{DF}_{2}, \mathrm{DF}_{7-9}\right)$. 
Table 2: Text example of design pattern cards

\section{Launch a Pilot Project}

What is it?

Pilot projects test a possible collaboration with a start-up for a specific period. Together, you estimate the feasibility, duration, and costs of a longerterm collaboration. Usually, existing products and services are tested on a smaller scale or newly developed.

\section{Where do I start?}

You have already gotten to know a promising start-up through other activities. Together, you now want to test out a possible collaboration. Agree on a form of collaboration with a realistic outcome that can be achieved in a shortterm pilot project.

How do I benefit?

- You get a quick and concrete result

- You probe the way of collaboration

- You get to know your potential partners better

Example from practice

AI and logistics: META-Regalbau and MotionMiners

- META-Regalbau is a renowned market leader for storage technology and solutions. To advance its digitalization, the SME became aware of

MotionMiners. The start-up had developed a technology in BETA phase to

analyze logistics processes in an industrial setup. The novel measurement method bases on artificial intelligence and learning sensors that collect relia-

ble data on internal workflows. Companies can thus identify their optimiza-

tion potential and efficiently increase work processes.

- In January 2018, both launched a four-month pilot study, analyzing process times for picking two items at a META plant. The test proved successful

work processes were accelerated and procedures for employees were less

stressful. Since then, both parties have been collaborating in ongoing

cooperation and working together as distribution partners.

- Note that a pilot project is always an important learning process for both

sides. META Regalbau first had to convince its workforce and the works

council. MotionMiners, on the other hand, was working with a technology

that was not yet fully mature, which sometimes led to disruptions in the operational process

Next, $D P_{O B 2}$ requires to only include the most relevant information in a standardized way to make different activities comparable and facilitate discussions. For this reason, we have iterated forms of presentation and information content during ADR cycles. As a result, we included features two to ten in the design. $\mathrm{DF}_{2}$ gives a quick overview of the expected costs. The costs correlate to the duration and intensity of the activity (see Figure 1). $\mathrm{DF}_{3}$ numbers the activities so that they can be quickly identified in context. This is supported by a different picture in each case $\left(\mathrm{DF}_{4}\right)$ since pictures are remembered faster than abstract signs, as well as a title $\left(\mathrm{DF}_{5}\right)$, which tries to describe the activity as selfexplanatory as possible. $\mathrm{DF}_{6}$ classifies the activity into a phase. The phase assignment is also coded in the background color. The activities are further characterized by employing a short description $\left(\mathrm{DF}_{7}\right)$. It is described how the activity is initialized $\left(\mathrm{DF}_{8}\right)$ and what benefits it brings to the $\mathrm{SME}\left(\mathrm{DF}_{9}\right)$. Additionally, $\mathrm{DF}_{10}$ contains a detailed description of an example of the practice. Table 2 provides a text example.

The first realization design principle $\left(D P_{R D I}\right)$ highlights the importance of a sufficient context for utilizing the EDA pattern cards. The key is to start with a clear goal in mind, pursue a big picture and define clear next steps. This is achieved by embedding it in a workshop format. A way to start is to describe a detailed success case (see example in Figure 4; $\mathrm{DF}_{11^{*}}$ ). Next, a strategic part aims to prioritize the SME's current challenges and thus provide a context for an EDA strategy (e.g., using a SWAT analysis; DF12*). Then, SME managers are asked to identify technologies, skills, and resources they need to solve the challenges previously identified. They are then requested to create an entrepreneur profile that has access to these resources $\left(\mathrm{DF}_{13^{*}}\right)$. This forms the foundation for utilizing the design pattern cards. In a further step, the SME managers should identify the resources available in the company from which the partner could benefit. This task highlights the collaborative nature of EDAs, as new ventures should be seen as equal partners rather than mere suppliers of missing resources. Finally, key next steps should be defined and the responsible entity for each step should be identified ( $\left.\mathrm{DF}_{14 *}\right)$.

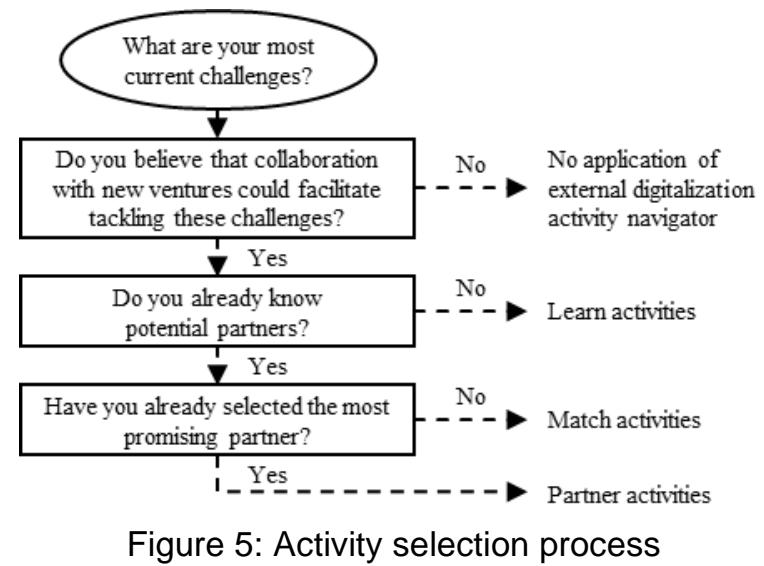

Further, $D P_{R D 2}$ states to reduce complexity and ensure clarity to ease ideation and help to choose suitable activities by preselecting design pattern cards. This is supported in particular by the decision logic $\left(\mathrm{DF}_{15 *}\right)$ illustrated in Figure 5. In line with Wrobel [35], we distinguish between three activity types: learn, match, and partner. First, by learning activities, SMEs and start-ups get to know each other and gain insights into the potential partner's values, goals, and challenges. The number of different actors can be relatively high. In contrast, the collaboration intensity is relatively low. Second, by match activities, SMEs and young ventures start collaborating over a longer time horizon to determine whether a long-term partnership is desired. Since match activities are more intensive, the number of different partners is significantly lower. Third, partner activities describe a medium to long time horizon. Collaboration intensity is high and thus the number of partners is likely to be small. The logic supports selecting the relevant activity cluster, which significantly reduces the number of design patterns to 
be evaluated by SME managers. After individual ideation, a group of business unit representatives can be asked to select the most promising activities and find a consensus. Finally, $D P_{R D 3}$ calls for building up emotional connections by showcasing empirical evidence to facilitate cultural change. This is achieved by the previously described introduction with an introductory case study $\left(\mathrm{DF}_{11 *}\right)$ and an analysis of the challenges $\left(\mathrm{DF}_{12 *}\right)$. In particular, however, this is achieved through the detailed description of a real-life example on an additional card for each activity $\left(\mathrm{DF}_{10}\right)$.

\subsection{Reflection and Learning}

We were able to identify 15 supporting attributes (features) of the navigation assistance for the successful maneuvering of SMEs through EDAs. Of these, 8 relate to the object and 9 to the realization design.

Concerning the object design, five SME managers mentioned that the pattern cards provide a comprehensive overview of the EDAs. The "dense overview" was highlighted as particular value-adding. The possibility to access 30 different activities was perceived as very interesting. One respondent emphasized the considerable educational value. Thus, the statements demonstrate adequate implementation of $D P_{O B I}$. However, the interviewees felt that the descriptions of the clustering of activities (learn, match, and partner) were not comprehensive enough. The description of the realworld collaboration example was also deemed insufficient to inspire by four SMEs. Based on the feedback, the description of the activity groups was expanded and the description of the examples was deepened by introducing a separate card (see $\left.\mathrm{DF}_{10}\right)$. According to six respondents, the amount of information provided within the design patterns and the level of detail was found useful. Four of them mentioned that the pattern cards are structured simply and straightforwardly, one noted, "what I like very much is this simple structure, it is very clear, and it is not too fancy, but describes directly what each activity means." However, one respondent noted that he had been overwhelmed with the variety of activities in the first step. All respondents agreed on the unified presentation of the activities and agreed that the pattern cards support discussions about EDAs. Further, the cards are seen as a source of inspiration as supported by the statement, "[w]ell, I actually think the idea of playing with the cards is fun and seeing what the possibilities are. If I want to work more with start-ups if I want to be inspired." Therefore, the DP2 is considered supported.

Regarding the realization design, six respondents found the definition and orientation of questions very helpful. That "the process and the methodology were clear and consistent" was stated by three respondents.
Moreover, the recording of the next steps is considered particularly important, but only if there is a certainty that this activity will also be pursued. At this point, $D P_{R D I}$ is considered implemented. Further, four interviewees stated that the workshop format helped to approach the complex issue and break it down clearly. In this context, one interviewee said: "I think the workshop picked up the topic well; it is short and concise and especially interesting for companies that still have little know-how of [EDAs] and collaboration possibilities. "With which the implementation of the $D P_{R D 2}$ is seen as given. In addition, the demonstration of the benefits by using a case study convinced the participants of the EDAs' usefulness. However, two interviewees mentioned that young companies are not always the best cooperation partners and that they could achieve better results with larger companies. Whereas, following two interviewees, this stat that the design pattern cards "allow for new inputs and discussions". Whereby "playing around" with the cards was noted as most exciting. While respondents did not mention the emotions, they felt while participating in the workshop, one interviewee pointed out, "for me, dealing with the topic was also a certain self-reflection about our organization and thus a valuable investment of time." Therefore, $D P_{R D 3}$ is also seen as supported. As a further point of condemnation, one interviewee noted, "it would be interesting for me to have a list of start-ups that are interested in such a cooperation. The effort for me to find them, I see as quite big." For that reason, a database of suitable new ventures that would be interested in collaborating would be complementary to the design pattern cards. This would significantly reduce the (starting) effort for SMEs.

\subsection{Formalization for Learning}

In the course of this ADR research approach, five design principles for EDA pattern cards were identified that ensure the greatest possible navigation in digitalization through alignment with external partners. Further, we demonstrated how 30 EDA pattern cards can be designed and implemented in a real-world SME context. As thus, the design principles presented include EDA navigator specifics based on the three hurdles previously identified: First, a lack of overall strategy and overview to prioritize needs is addressed through ease of access $\left(\mathrm{DP}_{\mathrm{OB} 1}\right)$ and standardized representation of EDAs in the form of pattern cards $\left(\mathrm{DP}_{\mathrm{OB} 2}\right)$ and orientation towards strategic goals $\left(\mathrm{DP}_{\mathrm{RD} 2}\right)$ and provision of a selection process for activities $\left(\mathrm{DP}_{\mathrm{RD} 1}\right)$. Second, the lack of digital technology skills and entrepreneurial spirit is addressed by focusing on open innovation activities (overarching strategy) and building up an emotional setup ( $\left.\mathrm{DP}_{\mathrm{RD} 3}\right)$. 
Third, a lack of collaborative culture considering the increasing complexity of digital ecosystems is also tackled by building emotional connections and illustration with practical evidence $\left(\mathrm{DP}_{\mathrm{RD} 3}\right)$ and considered by adhering to simplicity $\left(\mathrm{DP}_{\mathrm{OB} 2}, \mathrm{DP}_{\mathrm{RD} 2}\right)$.

\section{Conclusion and Outlook}

Our research aimed to develop and collect practice-oriented design knowledge for SME managers to select appropriate external digitization activities (EDAs) to improve their digital capabilities, considering limited human and financial resources. For this purpose, we followed an action design research (ADR) approach by Sein et al. [25]. To address the lack of navigational assistance, we came up with 30 EDAs presented as design pattern cards and derived two design principles for the object design (the actual card layout) and three for the realization design (the utilization of the cards in an SME context). Thus, we help practitioners and scholars alike in structuring and selecting EDAs for the digital transformation (DT) of SMEs. In particular, we provide a tool that helps SME managers gain an overview of EDAs, prioritize them, and place them in the overall strategy. Aligning activities to collaborate with new ventures helps SMEs tap into digital capabilities they do not yet possess. In addition, the activities can trigger a cultural change. E.g., attending meetups does not directly into new products or services, but it can stimulate an open culture of sharing. Here, integration into the digital ecosystem is essential to successfully embrace DT. In this way, we contribute to the concept of open innovation activities within the digital transformation literature of SMEs.

A number of limitations have to be considered with respect to our study. First, we gathered requirements from certain theoretical perspectives and experts. It might be possible that other areas of literature (e.g., dynamic capabilities or absorptive capacity) and practical perspectives have led to different results. Moreover, we were not yet able to fully evaluate the object and realization design. Therefore, we call for future research evaluating certain design features quantitatively. Further, we encourage future research that investigates the utility of specific ETAs based on the characteristics of the SME. We expect our overall research project to contribute a nascent design theory [46] to the artifact class of design pattern cards.

\section{References}

[1] S. Chanias and T. Hess, "Understanding Digital Transformation Strategy formation: Insights from Europe's Automotive Industry.," PACIS, vol. 296, 2016.
[2] M. Schmitz, C. Dietze, and C. Czarnecki, "Enabling digital transformation through robotic process automation at Deutsche Telekom," in Digitalization cases, Springer, 2019, pp. 15-33.

[3] J. Karimi and Z. Walter, "The Role of Dynamic Capabilities in Responding to Digital Disruption: A FactorBased Study of the Newspaper Industry," J. Manag. Inf. Syst., vol. 32, no. 1, pp. 39-81, Jan. 2015.

[4] P. Barthel and T. Hess, "Are Digital Transformation Projects Special?," in PACIS, 2019, p. 30.

[5] F. Wiesböck and T. Hess, "Digital innovations: Embedding in organizations," Electron. Mark., vol. 30, no. 1, pp. 75-86, Mar. 2020.

[6] G. Vial, "Understanding digital transformation: A review and a research agenda," J. Strateg. Inf. Syst., vol. 28, no. 2, pp. 118-144, Jun. 2019.

[7] L. Downes and P. Nunes, "Big bang disruption," Harv. Bus. Rev., pp. 44-56, 2013.

[8] C. Matt, T. Hess, and A. Benlian, "Digital Transformation Strategies,” Bus. Inf. Syst. Eng., vol. 57, no. 5, pp. 339-343, Oct. 2015.

[9] F. Abolhassan, Ed., The Drivers of Digital Transformation. Springer International Publishing, 2017.

[10] M. Cui and S. L. Pan, "Developing focal capabilities for e-commerce adoption: A resource orchestration perspective," Inf. Manage., vol. 52, no. 2, pp. 200-209, Mar. 2015.

[11] P. Kesting and F. Günzel-Jensen, "SMEs and new ventures need business model sophistication," Bus. Horiz., vol. 58, no. 3, pp. 285-293, 2015.

[12] C. Pelletier and L. M. Cloutier, "Challenges of digital transformation in SMEs: exploration of IT-related perceptions in a service ecosystem," 2019.

[13] S. Berghaus and A. Back, "Stages in Digital Business Transformation: Results of an Empirical Maturity Study.," in MCIS, 2016, p. 22.

[14] P. Neirotti, E. Raguseo, and E. Paolucci, "How SMEs develop ICT-based capabilities in response to their environment," J. Enterp. Inf. Manag., 2018.

[15] M. Levy, C. Loebbecke, \& P. Powell, "SMEs, co-opetition and knowledge sharing: the role of information systems," Eur. J. Inf. Syst., vol. 12, pp. 3-17, 2003.

[16] P. K. Banerjee and L. C. Ma, "Routinisation of B2B Ecommerce by small firms: A process perspective," Inf. Syst. Front., vol. 14, no. 5, pp. 1033-1046, Dec. 2012.

[17] H. Gimpel, S. Hosseini, R. X. R. Huber, L. Probst, M. Röglinger, and U. Faisst, "Structuring Digital Transformation: A Framework of Action Fields and its Application at ZEISS.," J Inf Technol Theory Appl, vol. 19, no. 1, p. 3, 2018.

[18] B. Barann, A. Hermann, A.-K. Cordes, F. Chasin, and J. Becker, "Supporting digital transformation in small and medium-sized enterprises: a procedure model involving publicly funded support units," 2019.

[19] S. Grama-Vigouroux, S. Saidi, A. Berthinier-Poncet, W. Vanhaverbeke, and A. Madanamoothoo, "From closed to open: A comparative stakeholder approach for developing open innovation activities in SMEs," $J$. Bus. Res., vol. 119, pp. 230-244, 2020.

[20] F. Rogo, L. Cricelli, and M. Grimaldi, "Assessing the performance of open innovation practices: A case study 
of a community of innovation," Technol. Soc., vol. 38, pp. 60-80, 2014.

[21] C. Gerling, M. Florian, D.de Paula, \& U. Falk, "Exploring Boundary Objects in Creative Projects: An Investigation of a Multi-Stakeholder Collaboration," 2020.

[22] G. Briscoe and C. Mulligan, "Digital innovation: The hackathon phenomenon," 2014.

[23] D. W. Kim, S. Trimi, S. G. Hong, and S. Lim, "Effects of co-creation on organizational performance of small and medium manufacturers," J. Bus. Res., vol. 109, pp. 574-584, Mar. 2020.

[24] A. R. Hevner, S. T. March, J. Park, and S. Ram, "Design science in information systems research," MIS $Q$., pp. 75-105, 2004.

[25] Sein, Henfridsson, Purao, Rossi, \& Lindgren, "Action Design Research,” MIS Q., vol. 35, no. 1, p. 37, 2011.

[26] J. E. van Aken, "Management Research Based on the Paradigm of the Design Sciences: The Quest for FieldTested and Grounded Technological Rules: Paradigm of the Design Sciences," J. Manag. Stud., vol. 41, no. 2, pp. 219-246, Feb. 2004.

[27] G. C. Kane, D. Palmer, A. N. Phillips, D. Kiron, N. Buckley, and others, "Strategy, not technology, drives digital transformation," MIT Sloan Manag. Rev. Deloitte Univ. Press, vol. 14, no. 1-25, 2015.

[28] K. Z. Zhou and F. Wu, "Technological capability, strategic flexibility, and product innovation," Strateg. Manag. J., 2009.

[29] D. Kiel, C. Arnold, \& K.-I. Voigt, "The influence of the Industrial Internet of Things on business models of established manufacturing companies. A business level perspective," Technovation, vol. 68, pp. 4-19, 2017.

[30] A. Singh and T. Hess, "How chief digital officers promote the digital transformation of their companies," in Strategic Information Management, 2020, pp. 202220.

[31] P. Barthel, C. M. Perrot, A. Benlian, T. Hess, and others, "Towards a Method for Evaluating Digital Innovation Projects," Darmstadt Technical University, Department of Business Administration ..., 2021.

[32] M. Andrade-Rojas, T. Saldanha, J. Khuntia, A. Kathuria, and W. F. Boh, "Overcoming Innovation Deficiencies in Mexico: Use of Open Innovation through IT and Closed Innovation through IT by Small and Medium Enterprises," presented at the Hawaii International Conference on System Sciences, 2021.

[33] H. Chesbrough, W. Vanhaverbeke, and J. West, "Open innovation," New Imperative Creat. Profit. Technol., vol. 1, 2006.

[34] F. Homfeldt, A. Rese, and F. Simon, "Suppliers versus start-ups: Where do better innovation ideas come from?," Res. Policy, vol. 48, pp. 1738-1757, 2019.

[35] M. Wrobel, K. Preiß, and T. Schildhauer, "Kooperationen zwischen Startups und Mittelstand. Learn. Match. Partner.," 2017.

[36] K. Lyytinen, Y. Yoo, and R. J. Boland Jr, "Digital product innovation within four classes of innovation networks," Inf. Syst. J., vol. 26, no. 1, pp. 47-75, 2016.

[37] Y. Yoo, R. J. Boland, K. Lyytinen, and A. Majchrzak, "Organizing for Innovation in the Digitized World," Organ. Sci., vol. 23, no. 5, pp. 1398-1408, Oct. 2012.
[38] S. Tumbas, N. Berente, and J. vom Brocke, "Digital innovation and institutional entrepreneurship: Chief Digital Officer perspectives of their emerging role," J. Inf. Technol., vol. 33, no. 3, pp. 188-202, 2018.

[39] C. Alexander, The timeless way of building. New York: Oxford University Press, 1979.

[40] M. Curley, J. Kenneally, and C. Ashurst, "Design Science and Design Patterns: A Rationale for the Application of Design-Patterns within Design Science Research to Accelerate Knowledge Discovery and Innovation Adoption," in Design Science: Perspectives from Europe, vol. 388, Springer International Publishing, 2013, pp. 29-37.

[41] C. Alexander, S. Ishikawa, and M. Silverstein, A pattern language: towns, buildings, construction. New York: Oxford University Press, 1977.

[42] X. Franch, C. Palomares, C. Quer, S. Renault, and F. De Lazzer, "A Metamodel for Software Requirement Patterns," in Requirements Engineering: Foundation for Software Quality, vol. 6182, R. Wieringa and A. Persson, Eds. Berlin: Springer, 2010.

[43] S. Petter, D. Khazanchi, and J. D. Murphy, "A design science based evaluation framework for patterns," ACM SIGMIS Database DATABASE Adv. Inf. Syst., vol. 41, no. 3, pp. 9-26, Aug. 2010.

[44] A. Osterwalder, Y. Pigneur, and T. Clark, Business model generation: a handbook for visionaries, game changers, and challengers. Hoboken, NJ: Wiley, 2010.

[45] A. Lucero and J. Arrasvuori, "PLEX Cards: a source of inspiration when designing for playfulness," in Proceedings of the 3rd International Conference on Fun and Games - Fun and Games '10, Leuven, Belgium, 2010, pp. 28-37.

[46] S. Gregor and A. R. Hevner, "Positioning and Presenting Design Science Research for Maximum Impact," MIS Q., vol. 37, no. 2, pp. 337-355, Feb. 2013.

[47] S. Gregor, L. Kruse, and S. Seidel, "Research Perspectives: The Anatomy of a Design Principle," J. Assoc. Inf. Syst., vol. 21, pp. 1622-1652, Nov. 2020.

[48] D. Jones and S. Gregor, "The Anatomy of a Design Theory," J. Assoc. Inf. Syst., vol. 8, pp. 312-335, 2007.

[49] J. Gläser and G. Laudel, Experteninterviews und qualitative Inhaltsanalyse als Instrumente rekonstruierender Untersuchungen, Wiesbaden: VS Verlag, 2010.

[50] C. Gerling, A. Bosch, D. de Paula, T. Haskamp, and F. Uebernickel, "Exploring Boundary Objects and their Affordances in the context of Design Thinking Projects from a Multi-Stakeholder Perspective," 2020.

[51] A. Leckel, S. Veilleux, and L. P. Dana, "Local open innovation: a means for public policy to increase collaboration for innovation in SMEs," Technol. Forecast. Soc. Change, vol. 153, p. 119891, 2020.

[52] R. Lisowska and R. Stanislawski, "The cooperation of small and medium-sized enterprises with business institutions in the context of open innovation," Procedia Econ. Finance, vol. 23, pp. 1273-1278, 2015.

[53] B. Bigliardi and F. Galati, "Which factors hinder the adoption of open innovation in SMEs?," Technol. Anal. Strateg. Manag., vol. 28, pp. 869-885, 2016. 